volgde hem in zijn lenen waaronder St.-Katerine op ${ }^{27}$. Merkwaardig is wel het feit dat deze Bouden in een oorkonde van 1381 als «miles» (ridder) wordt angeduid ${ }^{28}$.

Het is naar de naam van de twee eerste bezitters (Bouden of Boudewijn) dat het leengoed op St.-Katerine, op het einde van de $14^{\mathrm{e}}$ eeuw, de naam 's Heer Boudewijnsburg heeft gekregen, en de oude naam «ter Leyen» verdween. De hofstede met de feodale duiventoren, alsook de straatnamen «Leenhof» en «'s Heer Boudewijnsburg» op Assebroek herinneren er ons nog aan. Het is pas in 1828 dat het voormalige leengoed voor het eerst vermeld wordt als «de schaephofstede de Zeven Torrekens». Die naam berust echter op een vergissing. De feodale duiventoren, waarnaar de hofstede genoemd wordt, heeft in werkelijkheid acht zijtorentjes in plaats van zeven ${ }^{29}$.

J. SABBE

\title{
INVENTARIS VAN HET GOUD. EN ZILVERWERK IN APRIL 1578 TE BRUGGE OPGEEIST
}

$\mathrm{Na}$ de afzetting van Don Juan als landvoogd van de Nederlanden, werd aartshertog Matthias van Oostenrijk tot zijn opvolger erkend ( 8 dec. 1577). Zijn gezag was echter heel wat meer beperkt dan dat van zijn voorgangers. Niet alleen oefende de prins van Oranje een overwegende invloed op hem uit, maar ook de Staten-Generaal hadden een heel wat grotere bevoegdheid gekregen. In feite was het niet aartshertog Matthias die over de Nederlanden regeer-

27. Brussel, Alg. Rijksarchief, Rekenkamer, Reg. 1073, fol. 1.

28. Brugge, Rijksarchief, charters blaww, nr. 4661. Volgens J. Gailliard in zijn Bruges et le Franc, dl. 2, Brugge 1853, bl. 353, werd Bouden door de graaf tot ridder geslagen na de slag van Woumen tegen de Gentenaars in 1380. De auteur geeft geen bron op. Zijn gegevens over de familie de Vos in de $14^{\mathrm{e}}$ eeuw zijn verward en dikwijls foutief.

29. J. De Smet en H. Stalpaert, op. cit., bl. 62. 
de, maar het was veeleer de prins van Oranje, zoveel te meer daar de Raad van State hoofdzakelijk uit aanhangers van de prins was samengesteld. De grote invloed van Oranje werd trouwens al dadelijk officieel bekrachtigd door zijn benoeming, in januari 1578 , tot luitenant-generaal der Nederlanden.

Oorlogvoerende landen hebben immer met geldnood af te rekenen. Ook de opstandige Nederlanden, die in een oorlog met hun wettelijke vorst, koning Filips II van Spanje, waren gewikkeld, maakten op deze regel geen uitzondering.

Voor de regering van aartshertog Matthias was geldgebrek van den beginne af een knellende kwaal. Alle mogelijke bronnen om de staatskas te spijzen werden dan ook aangeboord; er werd zelfs overgegaan tot verplichte leningen en opeisingen.

Onder de vele maatregelen, die door de regering van Matthias werden genomen met het doel aan geld te geraken, willen wij hier vooral de aandacht vestigen op het plakkaat, dat op 16 februari 1578 te Antwerpen werd uitgevaardigd en waarbij het goud- en zilverwerk van de abdijen, de kloosters, de kerken, de hospitalen, de broederschappen, de gilden en de ambachten, alsmede van de steden en de kasselrijen werd opgeëist ${ }^{1}$. De gouden en zilveren voorwerpen zouden enkel en alleen aangewend worden als pand voor geldleningen en ze zouden na verloop van één jaar in ongeschonden toestand aan de eigenaars teruggegeven worden. Met de uitvoering van deze verordening in Vlaanderen werd Charles de l'Espinoy, lid van de Raad van Vlaanderen, gelast, bijgestaan door een adjunct, Jacob de Smet, van Brugge.

Wat inzonderheid Brugge betreft verzocht de l'Espinoy al dadelijk om de medewerking van het stadsbestuur. Maar hoezeer de commissaris ook mocht aandringen, toch ging de stadsregering niet op zijn verzoek in. Zij repliceerde niet ingelicht te zijn omtrent de «voet» waarop de opeisingen in de andere landsgedeelten zou geschieden en ze wenste derhalve eerst met de andere Leden van Vlaanderen de aange-

1. De tekst van dit plakkaat is gepubliceerd door A.C. De Schrevel, Comment disparut le trésor de la catbédrale de St.-Donatien à Bruges en 1578-1579, in Annales de la Société d'Emulation de Bruges, tom. LIX (1909), blz. 322-324. 
legenheid te bespreken. Pas nadien zou ze dienaangaande kunnen beslissen ${ }^{2}$.

Wij weten niet of de inlevering van de gouden en zilveren voorwerpen door de gezamenlijke Leden van Vlaanderen werd behandeld. In ieder geval zou de opeising worden doorgevoerd en was het Brugse stadsbestuur verplicht hieraan zijn medewerking te verlenen. Reeds op 27 februari werd door de magistraat beslist de heren Anselm de Boodt en Filips van Belle, respectievelijk schepen en pensionaris van de stad, te gelasten met het opmaken van de inventaris van de voorwerpen die voor inlevering in aanmerking kwamen. Maar al verleende de magistraat zijn medewerking, hij deed het met tegenzin en vol wantrouwen. Hij betwijfelde of het ingeleverde goud- en zilverwerk enkel en alleen tot pand zou aangewend worden en of het na verloop van één jaar aan de eigenaars zou teruggegeven worden. Integendeel vreesde hij, en terecht, de beroving en de vernietiging van de ingeleverde voorwerpen en stelde daarom als voorwaarde voor zijn medewerking dat het ingeleverde goud- en zilverwerk uitsluitend zou gebruikt worden voor doeleinden «ghepermitteirt by den ghescreven gheestelicken ende weirlicken rechte» ofwel in uiterste nood zou «gheëmploiert worden in subvencium pauperum» ${ }^{3}$.

Schepen de Boodt en pensionaris van Belle togen al dadelijk aan het werk om de opdracht, waarmede ze belast wa-

2. Brugge, Stadsarchief, secrete resolutiën van de Brugse stadsregering over de jaren 1575-1585, fol. 111 r.-v. : "Actum den XXV sporcle 1578. - Up 't vertooch van meester Charles Lespinoy, raedt 's Conyncx, van dat hy by commissie van der Conincklycke Majesteyt ghelast was te inventoriërene binnen deser stede alle de juweelen, vasselle, gout ende zelvere van der stede, de ghilden ende ambochten van diere metgaders de cloosters, capitels ende godshuusen, zo was denzelven commissaris ghegheven in antwoorde dat alzo die zake moste alomme gheëxploicteirt wesen up eenen voet, ende dat men niet en wiste up wat voet dat ghedaen zoude worden by den anderen leden, voor anderstont myn voorn. heeren den raedsheere daertoe eeneghe assistentie te doene men zoude tzelve tracteren tusschen den Leden ende daernaer doen als in rade zoude behooren».

3. Ib., t.a.p., fol. 111 v. : «Actum den XXVII februari 1578. Alsdan waren ghecommitteirt d'heer Ancelmus de Boodt, schepene, ende Philips van Belle, pensionaris, omme te bescryvene de juweelen van de kercken, cloosters ende capitelen deser stede ende versoucken die de wet gheleent te wordene, zo omme bewaert te zyne als omme ter noodt gheëmploieirt te wordene in subvencium pauperum ende andere usagen ghepermitteirt by den ghescreven gheestelicken ende weirlicken rechte. 
ren, ten uitvoer te brengen. $\mathrm{Zij}$ wendden zich tot de verschillende instellingen van de stad, die voor inlevering in aanmerking kwamen, met het verzoek om overlegging van de inventaris van hun gouden en zilveren voorwerpen. Aldus verschenen zij op 28 februari in het kapittel van de SintDonaaskathedraal, waar niet zonder bezwaar en na het maken van opmerkingen aan hun verzoek werd gevolg gegeven. De bezwaren, welke de kanunniken naar voren brachten, waren dezelfde die het stadsbestuur zelf tegenover commissaris de l'Espinoy had geopperd ${ }^{4}$.

Intussen was Charles de l'Espinoy wegens ziekte ontlast van zijn functie van commissaris en in deze hoedanigheid vervangen door Willem van Grysperre.

De overlegging van de inventaris van de gouden en zilveren voorwerpen betekende nog niet de inlevering van deze stukken. Pas op 16 maart werd de inlevering door het stadsbestuur bevolen. De ingeleverde voorwerpen zouden opgeborgen worden in de secreetkamer in de Halle, waar ook het stadszegel en de stedelijke privilegiën werden bewaard. De stadsthesaurier, Jan Breydel, en zijn klerk, Filips van Belle, werden meteen aangewezen om namens het stadsbestuur ontvangbewijzen in regel af te leveren «tot elcx gherusticheyt ende bewarenesse» 5 .

Op dezelfde dag beslistte de magistraat aan Cornelis van Hoghendorp, muntmeester te Brugge, uitdrukkelijk te verbieden de reserve aan muntstukken in enigermate te verminderen zonder speciale machtiging, alsook zilveren en gouden voorwerpen uit kloosters en kerken te smelten op straffe van «arbitraire correctie» en de waarde van de gesmolten voorwerpen te moeten uitkeren. Dezelfde straffen werden ook gepromulgeerd tegen de edelsmeden, die zich tot dezelfde praktijken zouden lenen ${ }^{6}$.

4. Zie: A.C. Schrevel, a.w., blz. 324.

5. Brugge, Stadsarchief, secrete resolutiën 1575-1585, fol. 115 v. : «Actum den XVI maerte 1578. - ... Item, was gheresolveert te doen overbrynghen de juweelen van de kercken, cloosters, ghilden ende ambochten ende dit al te bewarene in de secrete camere van der Halle daer rusten de zeghele ende privilegiën van der stede ende word tot ontfanghe ende receptie van dien ghecommitteirt d'heer Jan Breydele, thresorier-rentier, ende Philips van Belle, clerck van den thresorier, ende gheauthoriseert daervan quytantie ende recepisse te ghevene tot elcx gherusticheyt ende bewarenesse».

6. Ib., t.a.p., fol. 116: «Actum den XVI maerte 1578. - ... Item, was gheresolveirt te verbiedene Cornelis van Hoghendorp, munt- 
Reeds de volgende dag, 17 maart, schijnt het kapittel van de Sint-Donaaskathedraal aan het verzoek tot inlevering, dat door de stadsafgevaardigde Jan Breydel was gedaan, gevolg gegeven te hebben ${ }^{\text {? }}$.

Had de stadsregering tot dan toe slechts schoorvoetend de bevelen van het centraal bestuur uitgevoerd en zich steeds binnen de perken van de wettelijkheid gehouden, voortaan zouden de omstandigheden een andere wending nemen. Op 20 maart werd de stad door een bende Gentenaars, onder leiding van Frans de la Kethulle, overvallen en amper zes dagen later werd het stadsbestuur afgezet en door een nieuwe magistraat vervangen. Onnodig er op te wijzen dat het nieuwe stadsbestuur uitsluitend uit aanhangers van prins Willem van Oranje was samengesteld ${ }^{8}$. Van tegenstribbelen is er verder dan ook geen sprake meer : de bevelen van Oranje zullen voortaan getrouw opgevolgd worden.

Reeds op 29 maart kwam de inlevering van het goud- en zilverwerk opnieuw aan de orde van de stadsregering, die de volgende beslissingen trof. Het verzoek van commissaris Willem van Grysperre werd ingewilligd. Derhalve zouden «alle de juweelen ende ornamenten van de kercken, uuytghesteken van ghewijde zaken, metgaders ooc van ghilden ende ambachten» moeten ingeleverd worden. Als afgevaardigden van het stadsbestuur zouden de schepenen Jacob Broucqsault en Gislenus Kethele, alsmede Pieter Dominicle, lid van de kamer van de fortificatie, daarbij hun medewerking verlenen. Deze afgevaardigden zouden er niet alleen voor zorgen een getrouwe inventaris op te maken van de ingeleverde voorwerpen, met vermelding van het respectievelijke gewicint van de stukken, maar moesten ook nota nemen van de bezwaren welke door de gilden en de ambachten ter zake zouden geopperd worden alsmede van de moeilijkheden die de inlevering van deze waardevolle voorwerpen zouden opleveren. Instellingen of gemeenschappen, die bepaalde

meestre, zyn handen te ydelen van de pennynghen van de reserve van de munte zonder de wete van schepenen. Item, was gheresolveert te verbiedene denzelven muntmeestre ende voorts allen den goudtsmeden ende zelversmeden te smeltene eeneghe juwelen van de kercke up de peyne van de weirde van dien ende arbitraire correctie».

7. Zie: A.C. De Schrevel, a.w., blz. 327.

8. Zie : A.C. De Schrevel, Rectifications historiques II: Occupation de l'Ecluse - Surprise de la ville de Bruges par les Gantois Bruges sous la domination des gueux ..., in Annales de la Société d'Emulation de Bruges, tom. LXIX (1926), blz. 271-330. 
voorwerpen absoluut wensten te behouden, konden volstaan met de betaling in speciën van de tweederden van de waarde van het betrokken stuk. De inlevering zou gebeuren onder voorwaarde van restitutie, zoals door het plakkaat van 16 februari was voorgeschreven en zoals het ook was bepaald in de commissiebrief van de commissaris. Eén voorbehoud werd nochtans door de stadsregering geformuleerd. Mochten de andere steden, districten en bestuurlijke omschrijvingen niet tot een gelijkaardige opeising overgaan, dan behield de stad Brugge zich het recht voor de waarde van de ingeleverde voorwerpen af te trekken van de generale middelen en de beden, waarin de stad haar aandeel had af te dragen ${ }^{9}$.

De inleveringen begonnen op 9 april en hielden aan tot 28 april. De eerste gemeenschap, die aan de verordening tot inlevering gevolg gaf, was het hospitaal van Onze-LieveVrouw-ter-Potterie; het klooster van de paters Karmelieten kwam als nummer negentig het laatst aan de beurt.

Naast de afgevaardigden van het stadsbestuur, waren bij de inlevering aanwezig : de commissaris Willem van Grysperre en zijn adjunct Jacob de Smet, bijgestaan door de edelsmeden Adriaan Bultynck en Jan de la Tombe ${ }^{10}$.

Commissaris Willem van Grysperre beijverde zich om de

9. Brugge, Stadsarchief, secrete resolutiën 1575-1585, fol. 121 v.: «Actum den XXIX dach van maerte XVCLXXVIII. - Alsdan was by den college ter deliberatie van beede de bancken te consenterene meester Willem van Grysperre, advocat fiscael, als commissaris van den Hove, de lichtynghe van alle de juwelen ende ornementen van de kercken, uuytghesteken van ghewyde zaken, metgaders ooc van ghilden ende ambochten, wel verstaende ter kennesse van schepenen daertoe ghecommitteirt, te wetene: d'heeren Jacques Broucqsault ende Gheleyn Kethele metgaders Pieter Dominicle uuyter camere van de fortificatie ende behoudens aengaende de ghilden ende ambochten kennesse van de zwaricheden ende difficulteyten die daerup zullen moghen vallen ende dat van als ghemact worde goeden inventaris, note ende ghewichte, behoudens ooc dezelve juwelen te moghen lossen mids ghevende in ghelde de twee deelen van drien ende hendelick up de beloften van restitutie ghedreghen by de commissie ende van den commissaris ende indien d'ander landen ende steden niet laeten insghelycx lichten de juwelen van huerlieder districtie, dat 't college zal de weirde van dien moghen verhalen up de generale middelen van huerlieder dictrictie of andere beeden die daer loop zullen hebben».

10. Volgens de documenten op het Bisschoppelijk Archief was bij de eerste afgifte van de gouden en zilveren voorwerpen uit de SintDonaaskathedraal ook de edelsmid Jan Billiet aanwezig. Zie : A.C. De Schrevel, Comment disparut ..., blz. 331, 332. 
hem opgedragen taak zo vlug mogelijk af te handelen. Want alhoewel men op 28 april nog bezig was met inleveren, wegen en inventariseren van de voorwerpen en de complete inventaris van al de ingeleverde stukken pas op dezelfde dag ( 28 april) aan de stadsregering kon overgemaakt worden, eiste de commissaris dat het stadsbestuur reeds de dag te voren, 27 april, zou beslissen in de overbrenging van de ingeleverde voorwerpen naar Antwerpen, zetel van het centraal bestuur, toe te stemmen, hetgeen dan ook dadelijk na het beëindigen van de eigenlijke inlevering, op 28 april, kon geschieden ${ }^{11}$.

Hiermede was het eerste, echter niet het laatste bedrijf afgesloten waarbij heel wat kostbare voorwerpen voor altijd verloren gingen ${ }^{12}$. Kostbaar waren de ingeleverde voorwerpen niet alleen om hun intrinsieke waarde, maar vooral om hun uitzonderlijk belang voor de geschiedenis en de kunst. Want alhoewel anvankelijk uitdrukkelijk was bepaald, en later nog werd herhaald, dat de ingeleverde voorwerpen niet zouden vernietigd of gesmolten worden, doch enkel en alleen zouden worden aangewend als pand en dekking voor geldleningen, toch bleek al vroeg dat over hun bestemming geen twijfel bestond: ze zouden vast en zeker te gelde gemaakt worden ten einde de benarde toestand van de staatskas enigermate op te knappen.

Het tweede bedrijf begon op 25 september van hetzelfde jaar 1578. Toen besliste de magistraat de overige sieraden van de kerken, de kloosters en de andere instellingen van de stad, waaronder het nog overblijvende goud- en zilverwerk, op te eisen, ten einde deze waardevolle voorwerpen tegen plunderaars en soldateska te beveiligen ${ }^{12}$. Maar ook deze stukken keerden niet naar de eigenaars terug. Op 17 november 1578 werd door de stadsregering inderdaad beslist dat de aldus in bewaring genomen gouden en zilveren stuk-

11. Brugge, Stadsarchief, secrete resolutiën 1575-1585, fol. 129 : «Actum den XXVII aprilis 1578. - Alsdan was tot verzouke ende instancie van den advocat fiscal van Mechelen, meester Guillaume van Grysperre, gheconsenteert t'Hove ghevoert te wordene 't zelverwerck ende juwelen by denzelven advocat fiscal ghelicht van de kercken, ghilden, ambochten ende andere gheselschepen omme 's lands dienste gheëngagiert te wordene. - Dienvolghende zyn dezelve juwelen vervoert naer Antwerpen up den XXVIII dach aprilis 1578 volghende den inventaris rustende ter greffie».

12. De tekst van deze beslissing werd gepubliceerd in A.C. De Schrevel, Comment disparut ..., blz. 339-340. 
ken zouden «vercocht, vermunt of anderssins te ghelde ghebrocht» worden ${ }^{13}$.

Wat er tijdens deze tweede faze aan goud- en zilverwerk is teloor gegaan, schijnt eveneens aanzienlijk geweest te zijn, te oordelen althans naar de lijst van de stukken die door de Sint-Donaaskathedraal en de Sint-Salvatorskerk in oktober 1578 werden ingeleverd. Omtrent hetgene de SintDonaas toen heeft ingeleverd zijn wij goed ingelicht door de reeds herhaaldelijk aangehaalde publicatie van A.C. De Schrevel ${ }^{14}$; wat de Sint-Salvators aangaat vinden wij de lijst van de toen ingeleverde stukken achteraan in de rekening van de kerkfabriek over de periode van 1 januari tot 21 september 1578 :

«Zeker partye van zelvere juweelen toebehoorende deser kercke, overghelevert by deken ende kerckmeesters in de handen van die van der wet by laste van die van der wet gheweghen by Jaques de Cantere :

Eerst een cyborie daer men mede in processie gaet : VII marc VII oncen XV inghelschen.

Een glas met twee zelvere vergulde houcken : III marc VII oncen III inghelschen.

Twee cyborien : V marc VI oncen V inghelschen.

Twee ander cyborie: II marc III oncen.

Een zelver cruusken met glas daerinne : I marc IIII oncen VII inghelschen.

Een cruce van zelvere met hout, yser ende latoen : X marc III oncen.

Tsamen dese partien weghende eenendertich marck zeven oncen thyen ynghelschen aldus ghelevert up den XXIIII ${ }^{\text {en }}$ in octobre $\mathrm{XV}^{\mathrm{c}}$ achtenseventich in de handen van Pieter de Blende ende Christoffels de Ruddere, scepenen daertoe ghecomitteert by ghemeene college der stede van Brugghe» ${ }^{15}$.

13. Brugge, Stadsarchief, secrete resolutiën 1575-1585, fol. 198 : «Actum den XVII novembre 1578. - Alsdan was ghecomitteirt dheer Pieter de Blende, schepen, dheer Pieter Dominicle, thresorier, ende Heindryck Croes, notabele, omme te ghelde te brynghene 't zelvere van de cloosters, capittels ende andere kercken by den college ende tguene noch niet overbrocht en es in te doen commen ende overbrynghen in eersten omme ooc vercocht, vermunt of anderssins te ghelde ghebrocht te wordene stadsdienste».

14. Comment disparut ..., a.w., blz. 340-341.

15. Brugge, Bisschoppelijk Archief, rekeningen van de kerkfabriek van Sint-Salvators 1569-1585. 
Met de beslissing van de magistraat d.d. 17 november 1578 werd meteen het derde en laatste bedrijf in zake opeisingen van goud- en zilverwerk ingezet. Een ultieme poging werd inderdaad bevolen om de eventueel nog niet opgevorderde stukken op te sporen en in handen te krijgen ${ }^{16}$. Dientengevolge werd o.m. op 19 december 1578 aan de kerkfabriek van de Sint-Gillis bevel gegeven het nog resterende zilverwerk aan de stad over te leveren «daeronder oock begrepen de cyborie» ${ }^{17}$.

Zijn wij slechts fragmentarisch ingelicht over de voorwerpen die gedurende de tweede faze van de opeisingen werden ingeleverd, wat de derde faze betreft weten wij zogoed als niets. Daarentegen zijn wij voortreffelijk ingelicht over hetgeen tijdens het zogenaamde eerste bedrijf, in april 1578 , werd opgeëist en vernietigd. Wij bezitten inderdaad de complete inventaris van de toen ingeleverde voorwerpen.

Niettegenstaande het onbetwistbaar belang werd deze inventaris nog niet gepubliceerd. Enkel het gedeelte ervan, met betrekking tot de Sint-Donaaskathedraal, werd volgens besheiden uit het Bisschoppelijk Archief te Brugge uitgegeven en van een voortreffelijk commentaar voorzien door A.C. De Schrevel ${ }^{18}$. De hiernavolgende tekstuitgave van deze inventaris, volgens het exemplaar dat voor de Brugse stadsregering was bestemd, menen wij dan ook voldoende verantwoord.

A. SCHOUTEET

\section{INVENTARIS}

Inventaris van alle ende eenenyeghelicken de partien van juweelen van gout ende zelvere, respectivelicke overghebrocht zoo by de onfanghers van der abdien, priooren ende andere cloosters, midsgaders de kerckmeesters, ghildemeesters ende andere van der stede van Brugghe, ende onfaen by Jacques de Smet, als daertoe ghecommitteert by den hoochgheboren prince die erdtshertoghe van Oostenrycke, hertoghe van Bourgoingnen, gouverneur ende capitain general van den lande van herwaertsovere, by lettre patente van Zyne voorseide Hoocheit van den XVIen sporcle XVcLXXVIII in 't

16. Zie noot 13 hierboven.

17. Zie: E. Rembry, De bekende pastors van Sint-Gillis te Brugge, Brugge, 1890-1896, blz. 30.

18. Comment disparut ..., a.w., blz. 331-332. 
quaertier van Brugghe ende West-Vlaenderen, alle de voorseide partien gheweghen by Adriaen Bultynck ende Jan de la Tombe, zelversmeden, in de presentie van mynheeren den commissaris Grysperre ende zynen adjonct, ter date zo hiernaer volcht.

[Kanttekening:] Overbrocht den XXVIII dach van april 1578.

[1] 't Godtshuus van der Potterie in Brugghe.

Eerst een zelver cruuce up houdt besleghen, weghende metten ysere daeranne wesende IIII marcq VI onchen net ${ }^{1}$.

Twee remonstrantiën, tsamen weghende III marc VII inghelschen net.

Ende een paesbardt VI onchen min III inghelschen.

Overghebrocht den IX ${ }^{\text {en }}$ april 1578.

[2] 't Godtshuus van der Nazaretten.

Een remonstratie van zelver, weghende II marcq VII onchen en half.

Overghebrocht den $X^{\text {en }}$ april 1578.

[3] 't Godtshuus van Sincte-Juliens.

Een zelver hoofd van Sincte Juliens, weghende IIII marcq VI onchen net.

Twee ampullekens VIII onchen VIII inghelschen en half.

Een monstrance VII onchen XVII inghelschen en half.

Overghebrocht den $X^{\text {en }}$ april 1578.

[4] De ghilde van Sincte Quinten ten Magdaleene.

Twee cleene remonstransken ende een cleen Sincte Quintken IIII onchen XIIII inghelschen

Overghebrocht den $X^{\text {en }}$ april 1578.

[5] Sincte-Woulburghekercke.

Een zelver belle, weghende XII onchen.

Een zelver monstrantie, weghende XIX onchen en half. Item noch een cleene monstrantie, weghende XI onchen $\mathrm{V}$ inghelschen.

Item twee boucken besleghen met zelver voor ' $t$ beslach van desen boucken ende van cristalyne cruuce hiernaer verclaerst hebben de kerckmeesters over de redemptie gheleent een marcq zelvers, te XXXVIII s. par. d'onche, es II lb. X s. VIII d. gr., valent : XV lb. IIII s. tourn.

1. Het gewicht van goud en zilver wordt, zoals gebruikelijk, opgegeven in marken, onsen, engelsen en azen. 1 mark $=8$ onsen, 1 ons $=20$ engelsen, 1 engelse $=32$ azen. 1 mark $=246,076$ grammen. 
Item een processiecruuce van zelvere up hout besleghen ende met 't ysere daeran XI marcq IIII onchen.

Item noch een processiecruuce, besleghen ende met ysere als vooren, VII marcq VI onchen en half.

Item een zelver wieroockvat, weghende III marcq VII onchen.

Item twee zelver candelaers, binnen houdt zynde, weghende tsamen XIII marcq I onche.

Item drie zelver verghulde excels, wanof de platen achter lattoen zyn, met drie zelver verghulde cnoppens, weghende XII marcq V inghelschen.

Item een zelver verghulde cruuce III marcq IIII onchen.

Item een zelvere voet van eene cristalyne cruuce II marcq 5 onchen.

Item een zelver paesbardt VII onchen I inghelsche en half.

Item een zelver roede, binnen houdt, IIII marcq I onche V inghelschen.

Overghebrocht den XIen april XVcLXXVIII.

[6] Sinct-Jacobskercke.

Twee cruucen van zelvere up hout ende ysere XVIII marcq VI onchen.

Twee cantoristen van zelvere up houdt besleghen XI marcq III onchen.

Twee zelver wieroockvaten XI marcq III onchen.

Een zelver scelpe ende twee paesberden eensdeels verghult V marcq II onchen II inghelschen en half.

Twee zelver ampullekens II marcq II onchen V inghelschen.

Twee cleene cruuskens omme te zeynen XIIII onchen II inghelschen en half.

Een zelver quispele met houdt ende bustelen II marcq IIII onchen.

$$
\text { Overghebrocht XIen april } 1578 .
$$

[7] 't Baghynehof.

Twee wieroockvaten weghende IX marcq VI onchen XV inghelschen.

Item een zelver plateel IX onchen $\mathrm{X}$ inghelschen.

Item noch twee monstrantiën XII onchen min VII inghelschen en half.

Item noch een monstrantie dat den Berch heet II marcq VI onchen VIII inghelschen.

Item noch II ampullekens V onchen III inghelschen.

Item twee cruuskens up houdt ende yser V marcq IIII onchen; 
in stede van dezelve cruucen hebben die van den Wyngaerden of Baghynehof gheleent XXX s. gr., valent IX $\mathrm{lb}$. tourn.

Item een monstrantie van den Heleghen Cruus zonder cruus VII onchen $\mathrm{X}$ inghelschen.

Overghebrocht den XIen april 1578.

[8] Castaineboome.

Drie monstrantiën weghende tsamen II marcq V onchen IX inghelschen en half.

Overghebrocht XIen april XVcLXXVIII.

[9] Willemyne.

Een monstrantie weghende III onchen XIX inghelschen.

Overghebrocht XIen april 1578.

[10] Sincte Gillis.

Twee zelver inghels met zelver vlercken onder elck, met eene latoene plate, weghende tsamen XVIII marcq VI onchen en half.

Twee zelver candelaers XIIII marcq IIII onchen metten houtte.

Een zelver wieroockvat V marcq XVI inghelschen.

Twee ampullekens III onchen XVI inghelschen.

Een zelver quispele up houdt besleghen II marcq VI onchen. Overghebrocht XIen april 1578.

\section{[11] Sincte Claren.}

Twee cleene ampullekens IX onchen XI inghelschen.

Een zelver quispele up houdt besleghen II marcq II inghelschen en half.

Een cruuce up houdt besleghen $\mathrm{V}$ marcq net.

Overghebrocht XIen april 1578.

[12] Cellebroeders.

Een monstrantie zonder voet weghende XIII onchen XVI inghelschen.

Overghebrocht XIen april 1578.

[13] Sinte Obrechten.

Een zelver monstrantie ende een zelver paesbardt, weghende XI onchen II inghelschen en half.

Overghebrocht den XIIen april 1578.

[14] De ghilde van Sincte Marcq buuten Brugghe in de kercke van Sincte Cruus.

Een monstrantie van Sinte Marcq IIII marcq V onchen XV inghelschen. 
Een monstrantie van Sinte Pietre ende Pauwels XV onchen XII $1 / 2$ inghelschen.

Twee schalen weghende XVI onchen III inghelschen. Overghebrocht den XIIen april 1578.

[15] De ghilde van Sincte Cruus in Sinte-Cruuskercke.

Twee cruucen up houdt ende ysere tsamen IX marcq IIII onchen.

Noch een cruuce weghende, met hout daerinne wesende, XXIII onchen.

Ende twee ampullen, een paesbardt ende een brootvat II marcq net.

Overghebrocht den XIIen april LXXVIII.

[16] Sinte-Cruuskercke.

Een cruuce met houtte, besleghen met zelver ende isere, tsamen VIII marcq II onchen.

Een monstrantie van Sincte Catheryne, weghende zonder 't glas, XIII onchen XV inghelschen.

Noch een monstrantie weghende XIIII oncen.

Een zelver croes ende twee lepels, tsamen VI oncen.

[17] De ghilde van Sincte Anthuenis binnen Sinte-Cruuskercke.

Een platteel weghende IIII marcq III oncen.

Item noch drie trompetten weghende IX marcq IIII oncen $\mathrm{X}$ inghelschen.

Item noch een monstrantie IIII marcq 5 oncen $\mathrm{XV}$ inghelschen.

Item noch twee ampullen ende een paesbardt XII oncen min II inghelschen.

Ende noch een monstrantie weghende IIII marcq IIII oncen V inghelschen.

Overghebrocht XIIen april 78.

[18] 't Godtshuus van de Magdaleenen.

Een monstrantie van zelvere I marcq V onchen.

Noch een monstrantie VII onchen en half.

Item een wieroockvat met de scuppe ende 't lepelken III marcq IIII onchen.

Ende een cruuce up houdt besleghen metten ysere, II marcq.

Item den meestre van denzelven godtshuuse die heeft ghereduceert de voorscreven juweelen over de drie deelen van viere van de weerde XIIII lb. gr. den XIIen april 78, valent : IIIIxxIIII $\mathrm{lb}$. tourn.

[19] Bethaniën.

Twee ampullekens 8 onchen IIII inghelschen en half. 
Een monstrance VII onchen XII inghelschen en half.

Noch een cleen monstrance in de platse van twelcke die van den cloostre hebben gheleent XIII s. IIII d. gr. den XIIen april 78, valent : IIII lb. tourn.

[20] Sint Janshuus in Brugghe.

Twee cruucen van lattoen up houdt besleghen danof de Go zelver es van cleene improtance; waervooren den meestre van den hospitale gheleent heeft de somme van II $\mathrm{lb}$. gr. over de weerde van drie deelen van diere achtervolghende d'estimatie van den zelversmet, overghebrocht den XIIen april 1578, valent : XII lb. tournois.

[21] De ghilde van Sinct Sebastiaens.

Een zelver hooft van Sinct Sebastiaen in partie verghult, weghende VII marcq VII oncen.

Twee ampullen weghende II marcq.

Overghebrocht den XIIen april 1578.

\section{[22] Sincte Salvators.}

Twee zelver candelaers met waepens ende de stocken daerinne wesende XII marcq, af II marcq over de stocken, blyft X marcq net.

Noch II ander candelaers zonder stocken XII marcq I once.

Item twee groote monstrancen weghende met den reliquien ende glasen X marcq IIII onchen en half, af II marcq over de glasen, blyft VIII marcq IIII onchen en half.

Een zelver cruuce up houdt ende ysere IX marcq V inghelschen, of II marcq over 't houdt ende ysere, blyft VII marcq V inghelschen.

Een zelver sperwatervat weghende VIII marcq VII inghelschen.

Item 't upperste van twee canterstocken weghende VII marcq III onchen.

Een cruuce besleghen up houdt ende ysere met den langhen stock weghende tsamen IX marcq VI onchen en alf, af VI marcq VI onchen en half over 't hout ende ysere ende langhe stock, blyft III marcq net.

Item II ampullen XX onchen en half.

Een zelver belle XVII onchen en half.

Twee wieroockvaten weghende met de vullicheit daerinne wesende tsamen XII marcq III onchen en alf, af I marcq IIII onchen over de vullichede, blyft X marcq VII onchen en half.

Twee scelpen van zelvere XV onchen XII inghelschen.

Een zelver quispele III marck net, met yser ende bustelen af over tzelve II marcq, blyft I marcq net. 
Item III zelver plateelen wesende ongheteeckent zelvere, weghende VI marcq I alf onze.

Drie remonstranskens, de hoofdekens verghult, weghende tsamen met de glaskens ende relicxkens $\mathrm{XX}$ oncen schaers.

Alle welcke voorscreven partien de kerckmeesters van derzelver kercke hebben gheredimeert in ghelde voor de somme van hondert vierenveertich ponden grooten over de drie deelen van de weerde van diere te VI s. gr. d'onche, ghemerct dat al ongheteeckent zelver was al achtervolghende d'estimatie van den zelversmeden den XIIIIen april 78, valent de voors. somme in ponden tournois : VIIICLXIIII lb. tourn.

De ryve van Sincte Eloy wesende van houtte, besleghen met zelver platen toebehoorende 't comun der zelver kercke, ghestimeert by den zelversmeden de zelver platen weerdich te wesen ter somme van XVI lb. gr., waervooren die van den comune hebben gheleent de somme van XII lb. gr. over de drie deelen van viere van de voors. XVI lb. gr. ten daghe als boven, valent: LXXII lb. tourn.

[23] 't Ambocht van de huvetters.

Twee ampullekens $\mathrm{X}$ onchen en alf.

Een paesbardeken ende een brootvaetken XIIII onchen V inghelschen.

Ende II zelver cruucen up houdt ende ysere XXIII marcq II onchen.

$$
\text { Overghebrocht XIIII en april } 78 .
$$

[24] 't Ambocht van de scrynwerckers.

Twee cruucen up hout ende ysere XII marcq V inghelschen. Overghebrocht den XIIII'en april 78.

[25] De vleeschauwers.

Eenen zelveren kethene met eenen rynck VIII onchen min I inghelsche ende I quart.

Twee zelver cruucen up houdt ende ysere XXIII marcq IIII onchen.

Twee ampullekens XII onchen XI inghelschen.

Ende een zelver paesbardt ende een brootbusken II marcq V onchen $\mathrm{V}$ inghelschen.

Overghebrocht XIIIIen april LXXVIII.

[26] De muelenaers.

Twee zelver cruucen up houdt ende ysere weghende XXVI marcq I onche. 
Twee ampullekens VI onchen I halven inghelschen.

Ende twee monstrantiën IIII marcq I onche.

Overghebrocht XIIII en april 78.

[27] Droochscheerders.

Twee cruucen up houdt ende ysere XXIII marcq VI onchen.

Een brootvat ende een paesbart XII onchen net.

Een remonstrance IX onchen VIII inghelschen.

Overghebrocht XIIIIen april 78.

\section{[28] Cousceppers.}

Twee cruucen met houdt ende ysere $\mathrm{X}$ marcq III onchen net.

De cnoppen van de cruucen XV onchen.

Twee ampullekins VIII onchen IX inghelschen.

Ende een paesbardt ende een brootbusken IX onchen I inghelsche.

Overghebrocht XIIII en april 1578.

[29] Lootghieters.

Een monstransken met een kethelken weghende VI onchen en alf. Overghebrocht XIIII'n april 1578.

[30] Lysecleetwevers als Mandemakers.

Twee cruucen up hout ende ysere weghende XV marcq II onchen en half.

Twee ampullekens VI onchen VI inghelschen.

Ende noch een monstranstie XVIII onchen XVI inghelschen.

Overghebrocht XIIIIen april 78.

\section{[31] Tyckwevers.}

Twee cruucen up houdt ende ysere XIIII marcq XVI inghelschen. Ende een brootbusse IIII onchen V inghelschen.

Overghebrocht XIIIIen april 78.

[32] De ghilde van de Busschieters.

Die van de ghilde van Sincte Barble die de colveniers of busschieters houden binnen Brugghe overghebrocht hebbende de specificatie van huerlieder zelverwerck alsnoch onder hemlieden wesende ende naerdien up 't vertooch by hemlieden ghedaen ghecomuniquiert es gheweest met die van der wet der voors. stede van Brugghe es hemlieden gheconsenteert by den comissaris de redemptie van den voors. zelverewerck overschietende boven tghuene ghepant zynde an den hoofmeestre voor de somme van XXIII lb. gr. over de drie deelen van viere van de weerde van diere, welcke somme betaelt es by den hoofmeestre deken ende eedt den XIIIIen april 1578 , valent : IcXXXVIII lb. tourn. 
[33] Beeldemakers ende zadelaers.

Twee cruucen up hout ende ysere XVI marcq min II inghelschen. Een monstrantie ende een paesbardt XIII onchen net. Den deken (ende eedt die hebben gheredimeert de voors. monstrantie ende paesbardt den XVen april LXXVIII voor II lb. XVIII s. VI d. gr., valent : XVII lb. XI s. tourn.

[34] Sceppers.

Twee trompetten weghende VII marcq VII onchen XIIII inghelschen.

Overghebrocht den XIIIIen april 78.

[35] De fruteniers.

Twee zelver cruucen up houdt ende ysere tsamen weghende XI marcq VI onchen XV inghelschen.

Een paesbardt VIII onchen IX inghelschen.

Twee ampullekens IX onchen XVI inghelschen.

Een brootvatken III onchen net. In platse van dit brootvatken hebben deken ende eedt gheleent $X$ s. gr. den XIIIIen april LXXVIII, valent : III lb. tourn.

[36] De boghemakers.

Twee cruucen up houdt ende ysere weghende XVIII marcq VII onchen.

Overghebrocht XVen april LXXVIII.

\section{[37] Viscoopers.}

Twee zelver cruucen up houdt ende yser besleghen weghende tsamen XXIIII marcq X onchen.

Twee ampullen ende een monstransken, een plateelken ende een paesbardt tsamen V marck I onche XVI inghelschen.

Overghebrocht XVen april 1578.

[38] De ghilde van de $X^{m}$ martelaers in Onser-Vrauwenkercke te Brugghe.

Twee zelveren inghelen met' platen omme daerup te stane met een lanck stick zelvers, tsamen weghende IX marcq I onche X inghelschen.

Eenen zelveren aerm wesende in partie verghult weghende III marcq IIII onchen $\mathrm{X}$ inghelschen.

Een zelver monstrance weghende III marcq III onchen XIII inghelschen.

Twee zelver plateelen weghende IIII marcq II onchen en alf.

Een paesberdt III onchen XVI inghelschen en half.

Twee ampullen VI onchen VI inghelschen. 
Ende een brootbusse IIII onchen net.

Overghebrocht XVen april 78.

[39] De lynewevers.

Twee cruucen up houdt ende ysere XI marcq II onchen VIII inghelschen.

Een paesbart IIII onchen $\mathrm{V}$ inghelschen net. $15^{\text {en }}$ april 78.

[40] De wiltwerckers.

Twee zelver cruucen up houdt ende ysere XII marcq X inghelschen.

Een monstrantie weghende IX onchen XV inghelschen.

Overghebrocht $\mathrm{XV}$ en april 78.

[41] De verwers.

Twee cruusen up hout ende ysere XVII marcq V onchen net.

Twee ampullen XI onchen net.

Eenen quispele up houdt besleghen met zelver weghende tsamen II marcq II inghelschen en half.

Overghebrocht XVen april 78.

[42] De Cruuthalle.

Twee zelver cruusen up houdt ende ysere ende mette cnoppen XIIII marcq IIII onchen.

Twee zelver ampullen XVI onchen min XII inghelschen.

Een zelver platteel XIIII onchen IIII inghelschen.

Een zelver quispele metten houtte II marcq VI onchen.

Een zelver brootbusse ende een paesberdt tsamen XI onchen IX inghelschen.

Een zelver wieroockvat III marcq IIII onchen.

Een zelver scelpe ende een scuppe II marck II onchen VII inghelschen.

Een zelver monstrantie XIIII onchen XV inghelschen.

Overghebrocht XVen april LXXVIII.

[43] De cupers.

Twee cruusen met twee cnoopen up hout ende ysere XX marcq 1 onche.

Een zelver Sinte Lenaert IIII marcq IIII onchen XVII inghelschen en half.

Een zelver plateel II marcq IIII onchen.

Een paesbardt ende een brootbusse VIII onchen XIII inghelschen en half.

Overghebrocht XVen april 78. 
[44] De tegheldeckers.

Twee ampullekens ende een paesbardt midsgaders een brootvaetken III marcq I onche VII inghelschen.

Overghebrocht XVen april 78.

[45] De graeuwerckers.

Twee zelvere cruucen up hout ende isere XXII marck I once.

Item II zelver plateelen IIII marcq XV inghelschen.

Item II zelver cannekens XII onchen XIII inghelschen.

Item een zelver paesbardt IIII onchen IX inghelschen.

Item een zelver monstrantie weghende III marcq $\mathrm{X}$ inghelschen met 't latoen ende loot.

Overghebrocht XVen april 78.

[46] De ghilde van de spelieden.

Twee cruucen up houdt ende isere VII marcq III onchen V inghelschen.

Twee ampullekens $\mathrm{V}$ onchen XI inghelschen.

Een monstrantie IX onchen II inghelschen.

Noch een cleen monstrantie III onchen VI inghelschen.

Een zelver scale ende een lepele IX onchen min onderalven inghele.

Ende noch een paesbardt II onchen XI inghelschen.

Overghebrocht XVen april 1578.

[47] De winnebrooden.

Een zelver cruuce up houdt ende ysere IX marcq net.

Ende een monstrantie VII onchen I inghelsche.

Overghebrocht den XVen april 1578.

[48] De wynmeters.

Een cruuce weghende VII marcq VII onchen II inghelschen.

Een monstrantie weghende II marcq II onchen III inghelschen.

Overghebrocht den XVen april 78.

[49] De bezemakers.

Een cruuce up houdt ende isere weghende IX marcq VII onchen X inghelschen.

Een monstrantie van Sint Anne XV onchen V inghelschen.

Overghebrocht XVen april 78.

[50] De stoeldrayers.

Twee ampullekens VII onchen en half.

Een aerm van zelver $\mathrm{p}$ hout besleghen III marcq $\mathrm{V}$ inghelschen $\min$.

Overghebrocht $\mathrm{XV}^{\text {en }}$ april 78. 
[51] De ghilde van de Drie Sanctinnen.

Drie remonstrantiën van zelvere VII marcq III onchen VII inghelschen.

Noch een cleender monstransken weghende III onchen. De ghildebroeders hebben gheleent in 't ghesach van de voors. partie XVIII s. gr., valent: V lb. VIII s. tourn.

Ende twee ampullen II marcq VII inghelschen.

Overghebrocht XVen april 78.

[52] De lechwerckers.

Twee monstrantiën van zelver XI marcq IIII onchen XI inghelschen.

Twee ampullen ende een brootvaetken $\mathrm{X}$ onchen $\mathrm{XV}$ inghelschen. [53] De zuvelvercoopers.

Twee cruusen up houdt ende ysere XIIII marcq III onchen XV inghelschen.

Een plateel III marcq II onchen VII inghelschen en half.

Twee ampullen VI onchen I inghelsche en half.

Overghebrocht XVIen april 78.

[54] De backers.

Eenen zelveren verghulden cop weghende LIII onchen ende II inghelschen; drie zelver zoudtvaten XXXIII onchen XI inghelschen. Dese twee partien zyn ghelaten den gouverneur die altyts ghehadt hebbende loco pignoris. Dus alzo 't ghewichte excedeert van XI ghuldens de XXVII lb. X s. gr. van de t'achterheit, welcke XI ghuldens deken ende eedt ghefurniert hebben: XI lb. tourn.

Een zelver canne weghende VI marck IIII onchen XV inghelschen.

Drie zelver trompetten weghende XI marcq V onchen XIIII inghelschen.

Een zelver plateelken weghende II marcq I onche I inghelsche.

Twee zelver ampullekens, een paesbardt ende een brootvaetken weghende tsamen II marc III onchen XVII $1 \frac{2}{2}$ inghelschen.

Een monstrantie weghende II marcq XVII inghelschen en half.

Ende twee zelver cruucen weghende metten ysere XV marcq VI onchen.

Overghebrocht XVI ${ }^{e n}$ april 1578.

[55] De matsenaers.

Twee cruucen up houdt ende ysere XXII marcq V inghelschen.

Een brootvat VIII onchen VI inghelschen en half.

Ende twee ampullen ende een paesbardt II marcq V onchen V inghelschen.

Overghebrocht XVIen april 78. 
[56] Oudecleercoopers.

Twee cruusen up hout ende isere XIX marcq IIII onchen XV inghelschen.

Twee ampullekens VIII onchen onderalven inghel.

Overghebrocht XVIen april 78.

[57] Riemakers.

Twee cruucen up houdt ende zelver weghende XXV marcq IIII onchen.

Overghebrocht XVIen april 78.

[58] Hoemakers.

Twee cruusen up hout ende ysere XV marck V onchen VVII inghelschen en half.

Overghebrocht. XVIen april 78.

[59] De keersghieters.

Twee cruucen up hout ende ysere weghende XIIII marcq III onchen.

Twee ampullekens ende een brootvatken XIII onchen XVI inghelschen.

Overghebrocht XVIen april 78 .

[60] Handtschoewerckers.

Twee zelver cruucen up hout ende yser besleghen XIX marcq X inghelschen.

Ende een paesbardt VI onchen XVI inghelschen.

Overghebrocht XVIen april 78.

[61] Dobeerders.

Twee cruusen up hout ende ysere XV marcq IIII onchen.

Overghebrocht den XVIen april 78.

[62] Bardemakers.

Twee cruucen up hout ende ysere XXI marcq één onche.

Twee ampullekens ende een brootvatken XI onchen XV inghelschen.

Een paesbardt V onchen XVIII inghelschen.

Een monstrantie XIIII onchen II inghelschen.

Overghebrocht den XVIen april 78.

[63] Smeden.

Twee cruucen up hout ende ysere XXIII marcq net.

Twee ampullen ende een brootbusse midsgaders een paesbaerdt weghende tsamen één marcq VII onchen.

Overghebrocht den XVIen april LXXVIII. 
[64] Goudtsmeden.

Twee zelver cruusen up hout ende ysere XVII marcq III onchen net.

Een paesbardt $\mathrm{V}$ onchen $\mathrm{V}$ inghelschen.

Overghebrocht XVIen april 1578.

[65] Wapmakers.

Een cruuce up hout ende ysere VII marcq III onchen.

Twee ampullekens een paesbaerdt ende een brootvatken, tsamen weghende II marcq I onche.

Overghebrocht XVIen april 1578.

[66] De ghilde van Sinct Jacobs binnen Sinct-Jacobskerke.

Een cruuce up hout ende ysere VI marcq I onche X inghelschen. Overghebrocht XVIen april 78.

[67] De wullewevers.

Twee cruusen up houdt ende ysere XXXVIII marcq IIII onchen. Overghebrocht XVIen april 78.

[68] De vulders.

Twee cruucen up hout ende ysere XXIII marcq V onchen.

Overghebrocht XVIen april 78.

[69] Culstickers.

Twee cruusen up houdt ende ysere XVI marcq II onchen net.

Twee ampullen ende een paesbardt XII onchen XIIII inghelschen.

Twee trompetten ende een claroen XI marcq IIII onchen V inghelschen.

Overghebrocht XVIen april 78.

[70] Den Ouden Boghe in Brugghe.

Eenen zelveren gaey met eenen stock, een zelver bailliusroede up ysere, een brootbusse, tsamen weghende III marcq V onchen VII inghelschen.

Een goudt kethenken weghende een onche XIII inghelschen IIII azen.

$$
\text { Overghebrocht XVIen april } 78 .
$$

[71] De wynscrooders.

Een zelver brootvat, een zelver paesbart ende twee zelver ampullen weghende tsamen III marcq II $1 / 2$ oncen.

Overghebrocht XVII en april 78.

[72] De scippers.

Twee zelver ampullekens weghende VI onchen XV inghelschen. Overghebrocht den XVIIen april LXXVIII. 
[73] Scoemakers.

Twee ampullekens XII onchen XVI inghelschen.

Een brootvat IIII onchen XI inghelschen.

Een monstrantie IIII marcq II inghelschen en half.

Overghebrocht XVII en april 78.

[74] Scoeboutters.

Een zelver aerme VI onchen XIII inghelschen.

Een staende monstrantie van zelver II marcq IIII inghelschen.

Noch een ander monstrantie $\mathrm{V}$ onchen net.

Ende twee ampullekens weghende VI onchen min I inghelsche ende een quart.

Overghebrocht den XVIIen april 1578.

[75] De hoveniers.

Een monstrantie weghende XV onchen XII inghelschen en half.

Twee ampullen ende een brootbusse midsgaders een scaelken ende een paesberdeken XV onchen XVIII inghelschen en half.

Overghebrocht XVII en april 1578.

[76] Sincte Bazeliskercke.

Eenen cnoop van zelvere II marcq III onchen XVIII inghelschen.

Een brootbusse IIII onchen $\mathrm{X}$ inghelschen.

Twee ampullen VIII onchen IIII inghelschen.

Een paesberdt $\mathrm{X}$ onchen XV inghelschen.

Twee zelver candelaers $\mathrm{V}$ onchen VII inghelschen.

Twee zelver plateelen IIII marcq V onchen VI inghelschen.

Een wieroockvat V marcq II inghelschen.

Een scelpe met een lepelken VIII onchen net.

Een quispele up hout besleghen II marcq IIII onchen $\mathrm{X}$ inghelschen.

Een zelver pellicaen staende up eenen canterstock één marcq VII onchen I inghelsche $1 / 2$.

Overghebrocht XVIIen april $78^{1}$.

[77] De kercke van Sinte Cateryne buuten Brugghe.

Een wieroockvat weghende IIII marcq II onchen V inghelschen.

1. In verband met de relikwie van het $H$. Bloed had de stadsregering op 1 april beslist dat de kerkmeesters van Sint-Bazelis de «casse» en de «repositoire» mochten achterhouden. Zie : Brugge, Stadsarchief, secrete resolutiën 1575-1585, fol. 125: «Actum den eersten dach in aprilis 1578. - ... Alsdan was gheconsenteirt die van den gheselschepe van den Helighen Bloede in 't leveren van de juwelen van Sinte-Bazeliskercke achter te houdene de casse ende repositoire van den Helighen Bloede omme redenen schepenen daertoe moverende». 
Een monstrantie III marcq VI onchen IX inghelschen.

Twee zelver plateelen III marcq XVII inghelschen en half.

Twee ampullekens IX onchen VII inghelschen.

Een schale ende II lepels VIII onchen II inghelschen.

De kerckmeesters der zelver kercke hebben gheredimeert 't beslach van twee boucken voor II lb. gr. den XVIIen april 78, valent : XII lb. tourn.

[78] Brauwers.

Twee zelver cruucen up hout ende ysere XII marcq min VII inghelschen.

Twee zelver ampullen ende een brootvat I marcq VI onchen II inghelschen $1 / 2$.

Ende eenen zelveren cop met eenen decxele VI marcq III onchen IX inghelschen.

Overghebrocht XVII en april 78.

[79] Sinte-Annekercke.

Een zelver cruusken weghende XIIII onchen min IIII inghelschen.

Een ander cruusken weghende een marck IIII onchen VI inghelschen.

Een paesberdt VI onchen V inghelschen.

Een wieroockvat $\mathrm{V}$ marcq IIII onchen $1 / 2$.

Een wieroockscelpe VII onchen II inghelschen $1 / 2$.

Een zelver quispele metten houtte weghende II marcq III onchen III inghelschen $1 / 2$.

Ende twee ampullekens weghende I marcq I onche net. Overghebrocht XVII en april 1578.

[80] De ghilde van Onser Vrauwe van der Snee in Onser-Vrauwenkercke.

Twee zelver verghulde ampullen ende een brootbusse oock verghult tsamen II marcq III onchen. De ghildemeesters die hebben gheredimeert dezelve ampulle ende brootbusse voor IIII lb. gr. valent : XXIIII $\mathrm{lb}$. tourn.

Een zelver verghulde Onser Vrauwe zonder voet V marcq IIII onchen $\mathrm{X}$ inghelschen. De ghildemeesters die hebben gheredimeert dezelve Onser Vrauw voor X lb. gr. valent: LX lb. tourn.

Een zelver monstrantie weghende III marcq VII onchen XV inghelschen.

Overghebrocht XVIIen april 78.

[81] De thinnepotghieters.

Twee zelver ampullekens ende twee zelver Goden van cruucen 
weghende IIII marcq I onche net.

Overghebrocht XVII en april 78.

[82] Eechoutte.

Een zelver verghulde crotse metten stock zelver verghult wesende tsamen weghende $\mathrm{XV}$ marcq $\mathrm{V}$ onchen zuver.

Noch een cleene crootse met een stock weghende in zuiver zelver II marcq.

Twee ampullen weghende VI onchen $\mathrm{V}$ inghelschen.

Een zelver brootbusse weghende II onchen XIII inghelschen.

Ende twee zelver reliquien II marcq II onchen III inghelschen.

Alle welcke voorscreven partien zyn gheredimeert by den voorscreven prelaet over de drie deelen van IIII van de weerde up XV marck te II lb. X s. gr. 't marck bedraghende XXXIX lb. gr. welcke somme hy den XXIen april LXXVIII ghefurniert heeft, valent : IIcXXXIIII lb. tourn.

[83] De Graeubroeders.

Een cruuce van zelver weghende II marcq I onche VI inghelschen.

Twee zelver ampullen weghende VI onchen $\mathrm{X}$ inghelschen.

Overghebrocht XVII en april 78.

[84] De ghilde van Sinct Anthuenis in Sint-Jacobskercke.

Twee monstrantien weghende XIII onchen XIII inghelschen en half.

Overghebrocht XXIen april 1578.

[85] De ghilde van de Magdaleene in de kercke ten Magdalenen buuten Brugghe.

Een Magdaleene van zelver weghende XXXVII onchen en half.

Twee cleene monstranstien ende een paes paesbardt tsamen weghende $\mathrm{V}$ marcq.

Alle welcke voorscreve partien zyn gheredimeert by de ghildemeesters der voors. ghilde over de III deelen van viere van de weerde voor de somme van VIII lb. VI s. VIII d. gr. den XXIen april XVcLXXVIII, valent : $\mathrm{L} \mathrm{Ib}$, tourn.

[86] De Predicaren in Brugghe.

Een zelveren verghulde cruuce met eenen voet van tzelve, weghende zuver II marcq in zelver.

Twee zelver candelaren weghende zuver in zelver VIII marcq III onchen.

Een wieroockvat weghende zuver in zelver IIII marcq IIII onchen. 
Ende twee cruucen up hout wanof tzelve weecht I marck blyft zuvers XI marcq.

Ende hebben gheleent die van de Predicaren voorseit XXVI $\mathrm{lb}$. gr. over de drie deelen van viere van de weerde van alle de partien, valent: CLVI lb. tourn.

[87] De meerseniers.

Eenen zelveren aerme weghende III marcq net.

Noch een monstrantie wesende eenen Sincte Niclaeus IIII oncen net.

Een zelver Onser Vrauwe zonder voet II marcq III onchen net.

Een zelver Sinct Jooris III marcq XII inghelschen $1 / 2$.

Item IIII zelver ampullekens III marcq II onchen.

Een zelveren Sinct Anthuenis weghende V onchen X inghelschen.

Item een monstrantie boven met eenen Sinte Nicolaeus weghende zonder relisuie ende ghelas een marcq IIII onchen min II inghel schen.

Item noch een monstrantie met een cruuc boven II marcq min $\mathrm{X}$ inghelschen.

Item een brootbusse V onchen VIII inghelschen.

Noch twee zelver platteelen IIII marcq III onchen XV inghelschen.

Item een zelver cruuce ende noch twee cruucen up houdt, dewelcke der neerynghe zyn ghelaten voor twee marcq zelvers.

Alle welcke voorscreven partien den deken ende die van der neerynghe voorseit hebben gheleent in ghelde de somme van XLIIII lb. VI s. VIII d. gr. ende dat over de XVII marcq IIII onchen wesende de III deelen van viere te VI s. IIII d. gr. d'onche, valent de voors. somme den XXII en april LXXVIII : IIcLXVI lb. tourn.

[88] Sincte Donaes.

Een schoone costelicke ryve van Sincte Donaes van zelver we ghende IIcXXVII marcq X inghelschen.

Een fiertre van Sinte Basilius Magnus weghende XXVIII marcq VI onchen VII inghelschen $1 / 2$.

Een hooft van Sinct Jans Baptiste weghende XXI marcq III onchen VIII inghelschen.

Item het hooft van Sinct Jans Chrisostomus weghende XXVIII marcq VII onchen V inghelschen.

Item het hooft van Sincte Pieters meestdeel verghult met de schouders ende een pontificatiecroone ende met diveersche bellekens ende IIII leeukens weghende tsamen XXXIX marcq V onchen $\mathrm{XV}$ inghelschen.

Item eenen voet van houtte besleghen met zelver platen daer het 
voorscreven hooft up staet weghende met het hout XXXVII marcq IIII onchen ende by estimatie van den zelversmidt XVI marcq III onchen II inghelschen.

Item een zelver verghulde beelde van Onser Vrauwe weghende $\mathrm{X}$ marcq IIII onchen XVII inghelschen $1 / 2$.

Item een zelver verghulde beelde van Sint Donaes VII marcq II onchen III inghelschen.

Item een monstrantie van zelver verghult met een doorne van Onsen Heere croone III marcq V onchen III inghelschen.

Item een aerme van zelver verghult met de reliquien van Sincte Machuut VIII marcq VII onchen XVII inghelschen.

Item een monstrantie van zelver verghult met diveersche reliquien II marcq IIII onchen XVII $1 / 2$ inghelschen.

Item een schoone beelde van Sint Jeronimus met eenen leeuwe XIIII marc I onche V inghelschen.

Item een zelvere verghulde beelde van Sincte Catheryne IIII marcq $\mathrm{V}$ onchen $\mathrm{XV}$ inghelschen.

Overghebrocht den zessentwyntichsten dach van april XVcLXXVIII ${ }^{1}$.

[89] Onser Vrauwe.

Eerst een beelde van Sinct Jan weghende XXV marcq III onchen. Item een beelde van Onser Vrauwe weghende XXIII marcq VIII inghelschen.

De beelde van Sincte Bonefacius weghende XVII marcq net.

Noch een beelde van Onser Vrauwe weghende XII marcq VII onchen $\mathrm{X}$ inghelschen.

Twee wieroockvaten IX marcq $\mathrm{V}$ onchen.

De kerckmeesters derzelver kercke hebben gheredimeert de V bovenghescreven partien over de redemptie van de drie deelen van viere van de weerde bedraghende in zelver LXIIII marcq VI inghelschen compt in ghelde hondert drie ende vichtich ponden XIII s. gr., valent : IXcXXI $\mathrm{lb}$. XVIII s. tourn.

Twee zelver platteelen weghende 5 marcq III onchen XV inghel. schen.

Een sperrewatervat weghende VIII marcq I onche.

't Zelver van een crucefix IIII marcq IIII onchen.

1. Het kapittel van de Sint-Donaaskerk leverde haar voorwerpen in op 10 en 11 april. Blijkbaar hadden de kanunniken gepoogd een en ander stuk te ontduiken, want op 18 april eiste commissaris Willem van Grysperre ook de inlevering van de ontbrekende voorwerpen. (Zie A.C. De Schrevel, Comment disparut ..., blz. 10-12).) Aldus is het wellicht te verklaren waarom in onze inventaris de inlevering pas op 26 april staat opgetekend. 
Twee conterstocken weghende VII marcq V onchen $1 / 2$.

Een zelver belle III marcq III onchen V inghelschen.

Twee ampullen weghende II marcq IIII onchen.

Twee monstrantien daermede men omghaet III marcq I onche XII inghelschen $1 / 2$.

Een brootbusse weghende VII onchen X inghelschen.

d'Apostelen van den hooghen hautaer weghende XXIIII marcq net.

De fiertre van Sinte Bonefacius weghende in zelver CLII marcq III onchen XV inghelschen.

Twee zelver candelaers XIIII marcq III onchen.

Een zelver quispelle up hout II marcq II onchen.

Alle welcke partien met oock de somme van ghelde hiervooren verclaerst ghefurniert es den XXVIen april LXXVIII.

\section{[90] Carmers.}

Twee zelver candelaers VIII marcq $\mathrm{X}$ inghelschen.

Twee zelver wieroockvaten $\mathrm{X}$ marcq $\mathrm{V}$ onchen.

IIII zelver ampullekens II marcq IIII onchen.

Twee zelver cruusen up houdt ende yser by estimatie VI marcq.

Een zelver quispel ende een paesbardt III marcq II onchen.

Alle welcke voorseide partien den prioor van den voors. convente van den Carmers heeft gheredimeert voor de somme van XLVIII lb. gr. Vlaems ende dat over de drie deelen van viere van de weerde den XXVIII en april 78 , valent : IIcIIIIxxVIII lb. tourn.

De partien van zelverwerck in desen inventaris gheroert midtsgaders de sommen van ghelt daerinne ghementionneert zyn respective ontfaen by my Jacques de Smet daertoe ghecommitteert zoo hiervooren in 't prohemie verclaert es naerdien die gheweghen zyn gheweest ende gheëstimeert ter presentie van den commissaris ende adjoinct omme daerof de overleveringhe te doene an den tresorier generael van de Staten Dieryc vander Beke volghende d'ordonnantie van Zyne Hoocheyt. Des t'oorconde desen gheteekent den XXVIIen april XVcLXXVIII.

$$
\text { (Get.) J. De Smet. }
$$

Ick commissaris onderscreven attestere geen andere partien van selvere noch in gereeden geldt by den voorn. ontfanger ontfaen te zyne van degene gespecifieert in desen inventaris gewegen ende geëstimeert respective t'onser presentie.

(Get.) G. van Grysperre.

Brugge, Stadsarchief, Brieven en Akten, 1570-1579. Op de rugzijde van het laatste blad: «Inventaris van zelver ghesonden uuyter stede van Brugghe t'Hove». 


\section{Lijst van de instellingen die edelsmeedwerk bebben ingeleverd}

NB. De nummers verwijzen naar de paragrafen.

A. Ambacbten :

Baardmakers 54

Bakkers 62

Beeldenmakers en zadelmakers 33

Bezemmakers 49

Boogmakers 36

Brouwers 78

Dobbeerders 61

Droogscheerders 27

Huidevetters 23

Fruiteniers 35

Goud- en zilversmeden 64

Grauwwerkers 45

Handschoenwerkers 60

Hoedenmakers 58

Hoveniers 75

Kaarsengieters 59

Koussceppers 28

Kruidhalle 42

Kuipers 43

Kulktstikkers 69

Legwerkers 52

Lijnwevers 39

Lissekleeswevers en mandenmakers 30

Loodgieters 29

Meerceniers 87

Metselaars 55

Molenaars 26

Oudkleerverkopers 56

Riemmakers 57

Scheppers 34

Schippers 72

Scoenbouters 74

Schrijnwerkers 24
Smeden 63

Speellieden 46

Stoeldraaiers 50

Tegeldekkers 44

Tijkewevers 31

Tingieters 81

Ververs 41

Visverkopers 37

Vleeshouwers 25

Vulders 68

Wapenmakers 65

Wijnmeters 48

Wildwerkers 40

Winnebroden 47

Wolwevers 67

Wijnschroders 71

Zuivelverkopers 53

B. Broederschappen :

van O.L.Vrouw ter Sneeuw

in de O.L.Vrouwekerk 80

van Sint-Antonius in de

Sint-Jacobskerk 84

van het $\mathrm{H}$. Kruis in de Sint

Kruiskerk 15

van Sint-Antonius in de

Sint-Kruiskerk 17

van Sint-Jacob in de Sint-

Jacobskerk 66

van Sinte-Magdalena in de

Sinte-Magdalenakerk 85

van Sint-Marcus buiten de

stadswallen 14

van Sint-Quinten in de Sinte-

Magdalenakerk 4

van de Tienduizend Mar- 
telaren in de O.L.Vrouwekerk 38

C. Kloosters :

Begijnhof 7

Bethanië 19

Cellebroeders 12

Eekhoute 82

Grauwbroeders 83

Karmelieten 90

Kastanjeboom 8

Magdalenahospitaal 18

Nazareth 2

Onze Lieve Vrouw ter Potterie 1

Predikheren 86

Sint-Clara 11

Sint-Janshospitaal 20

Sint-Juliaan 3

Sint-Obrecht 13

Wilhelmijnen 9
D. Parocbiekerken en kapellen :

Onze-Lieve-Vrouw 89

Sint-Anna 79

Sint-Bazelis of Heilig

Bloed 76

Sint-Catharina 77

Sint-Kruis 16

Sint-Donaas 88

Sint-Gillis 10

Sint-Jacobs 6

Sint-Salvators 22

Sint-Walburga 5

E. Rederijkersgild:

Drie Santinnen 51

F. Schuttersgilden:

Busseschieters 32

Sint-Joris (Oud Hof) 70

Sint-Sebastiaan 21 